\title{
On the trail of the neutrino
}

\author{
Huge arrays of \\ detectors now have
}

these ghostly particles in their sights - but will what they see lead physicists to rethink the standard model? Dan Falk investigates.

$n$ $\mathrm{n}$ the subatomic zoo, few particles are as elusive as the neutrino. The Universe is awash with them, but they slide through most forms of matter with ease. Billions have passed through your body since you started reading this article. By one estimate, the average neutrino could travel for 1,000 light-years through solid matter before being stopped.

This reluctance to interact with matter makes neutrinos difficult to detect. But, almost half a century after they were first spotted, neutrinos have become the focus of intense study. Exciting results from existing detectors, together with plans for new detector projects, promise to make this a busy decade for neutrino hunters.

The Sudbury Neutrino Observatory (SNO) in Ontario is poised to illuminate the physics of neutrinos produced by the Sun, and a new detector in Antarctica is making progress towards studying neutrinos from deep space — a first step towards building a larger astrophysical neutrino observatory. Other detectors in North America, Europe and Japan are catching neutrinos produced when cosmic rays hit the atmosphere or by particle accelerators here on Earth. "I think it's fair to say that this is the era of the neutrino," says John Bahcall of the Institute for Advanced Study in Princeton, New Jersey, a pioneer of neutrino physics.

\section{Three of a kind}

According to the standard model, the successful but incomplete theory that describes fundamental particles and their interactions, neutrinos come in three types or 'flavours': electron, muon and tau neutrinos. The names derive from links within the model between the neutrinos and other particles - the familiar electron and its rarer, heavier cousins the muon and tau

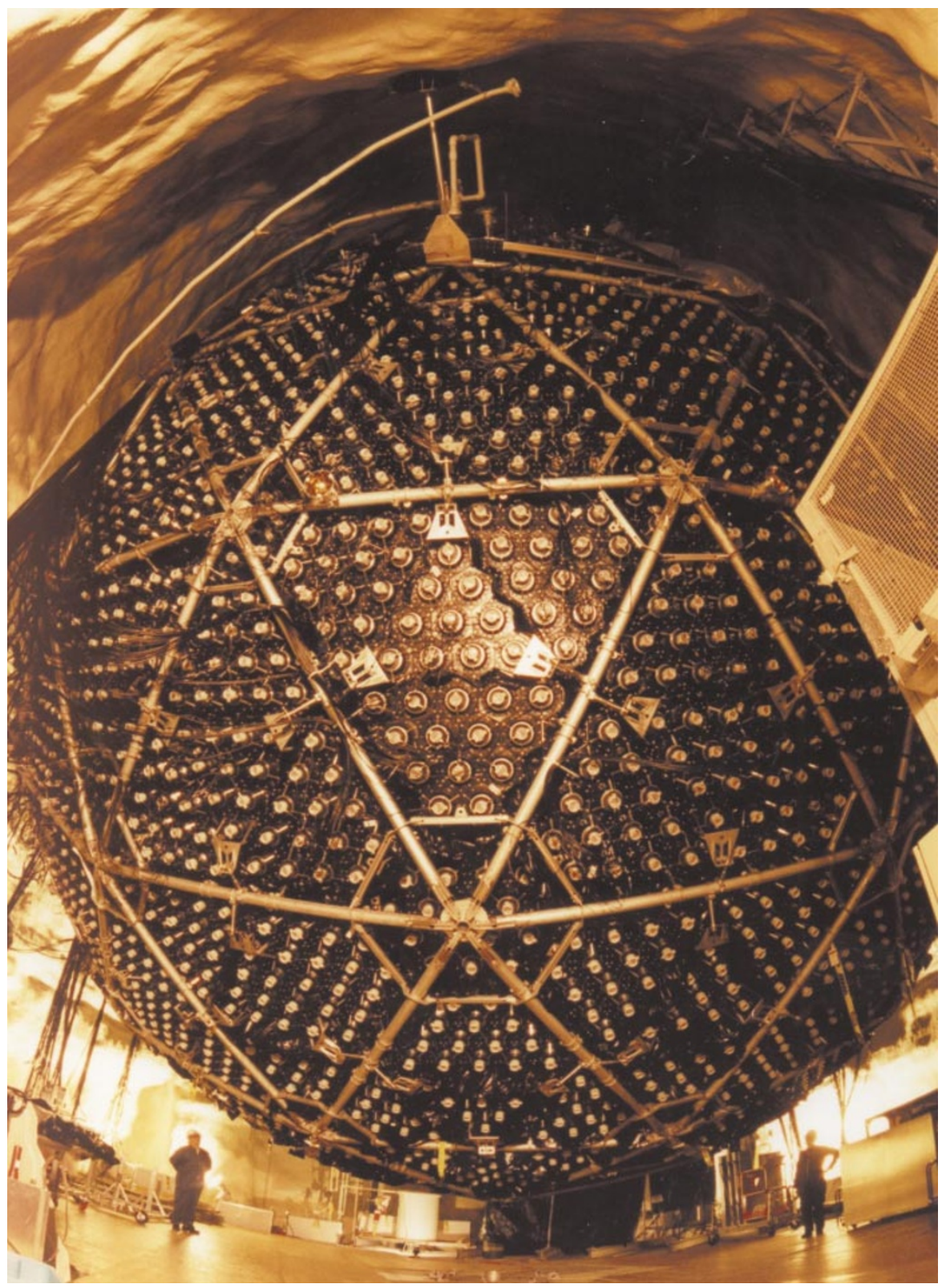

Full house: the detector at Sudbury Neutrino Observatory can detect all three flavours of neutrino.

particles. The last of the three flavours to be detected was the tau neutrino, finally snared last year in an experiment at the Tevatron accelerator in Fermilab, near Chicago ${ }^{1}$.

The problem faced by neutrino hunters is that all three flavours of neutrinos are extremely unwilling to interact with other subatomic particles. Of the four types of fundamental interaction between particles, neutrinos interact almost exclusively through the weak nuclear force, the puny force responsible for some radioactive decays. As they do not carry charge, neutrinos avoid electromagnetic interactions. Nor do they interact through the strong nuclear force that binds nuclei together. The jury is still out on whether neutrinos feel gravity, but if they do, the forces involved will be tiny.

So the only practical way to snag a neutrino hinges on the weak nuclear force and the interactions between neutrinos and protons, neutrons or electrons it governs. Physicists maximize their chances of observing these rare interactions by monitoring huge numbers of potential neutrino targets. Large tanks of water are a common choice - water is cheap, plentiful and, being transparent, it allows physicists to spot any interactions that occur deep inside the tank. The tanks are held deep below ground, so that other particles are screened out by the overlying rock.

There are several types of interaction to look for. The simplest is 'elastic scattering' high-speed collisions between electrons in water molecules and incoming neutrinos. The collision leaves the electrons travelling faster than the speed of light through water. As a result, the electrons emit dim, blue flashes of light known as Cerenkov radiation the optical equivalent of the 'sonic boom' created by supersonic aircraft. Neutrinos can also collide with neutrons or protons in 
atomic nuclei, and some of the particles produced in these reactions also travel fast enough to produce Cerenkov radiation. In each case, the flashes of light can be captured by photomultiplier tubes (PMTs) on the sides of the detector tanks.

Detectors such as these could help to settle the most pressing issue in neutrino physics - that of the neutrino mass. The standard model says that neutrinos should have zero mass, but evidence from solar physics and some proposed Grand Unified Theories efforts to unify electromagnetism and the strong and weak nuclear forces as different aspects of a single interaction - points instead to the particles having a small mass. For physicists, the tell-tale sign that neutrinos have mass would be 'flavour-switching', or oscillation. Neutrinos oscillate if they switch from one flavour to another as they travel. The way in which such switching would occur is not fully understood, but physicists know that to oscillate neutrinos need to have mass ${ }^{2}$.

In 1998, a team of US and Japanese researchers using the Super-Kamiokande, or Super-K, detector in Japan found evidence that appeared to support the idea of neutrinos with mass $^{3}$. The story landed on the front page of The New York Times under a headline declaring that the "Universe May Never Be the Same".

\section{Something in the air}

Buried deep under Mount Ikena near the town of Kamioka, 200 kilometres northwest of Tokyo, the Super-K detector uses 13,000 PMTs to monitor a 50-million-litre tank of water. It searches for signatures of 'atmospheric neutrinos' - electron or muon neutrinos produced when high-speed atomic nuclei and electrons from deep space collide with oxygen or nitrogen atoms in the Earth's atmosphere. These neutrinos can be singled out because they come from a different direction and have a different energy compared with other neutrinos.

The present theory of how atmospheric neutrinos are generated suggests that Super$\mathrm{K}$ should detect twice as many muon neutrinos as electron neutrinos. Instead, it is recording roughly equal numbers of each ${ }^{3}$. This, argues the Super-K team, shows that some of the muon neutrinos are turning into something else - tau neutrinos - as they travel through the atmosphere and the Earth's crust. Super-K cannot detect tau neutrinos because the particles produced when they collide with atomic nuclei are usually too short-lived to generate Cerenkov radiation.

Although the Super-K results cannot give a specific value for the mass of any neutrino flavour, they can be used to calculate a mass difference - in this case, a fraction of a millionth of the mass of an electron - between the muon and tau types. In other words, either the muon or the tau neutrino must have a mass at least this large.

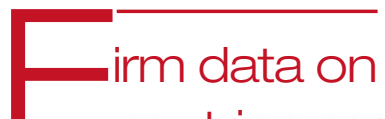

neutrino mass

\section{could point the way to} a more elegant theory.

But the experiment is not foolproof. The calculation depends on the rate at which atmospheric neutrinos are produced, and this is not known precisely. Those involved with the project are confident that Super-K has indeed seen neutrino flavour-switching. "The oscillation theory quite nicely explains our data," says Yoji Totsuka, the observatory's director and head of the Institute for Cosmic Ray Research at the University of Tokyo.

\section{Solar flaws}

Conclusive evidence for neutrino oscillation would also solve one of the longeststanding questions in solar physics. From what we understand about how the Sun produces neutrinos, 60 billion solar neutrinos should pass through every square centimetre of the Earth's surface every second. But measurements from neutrino detectors suggest that only between a half and a third of this figure are reaching the Earth ${ }^{2,4}$.

Either the models that describe the Sun's interior are flawed - and most scientists doubt this to be the case - or something is happening to the neutrinos between their emission from the Sun's core and their arrival on Earth. In the main, the Sun generates electron neutrinos, but most physicists think that many of these switch flavours en route, turning into muon neutrinos or tau neutrinos. Neutrino detectors, including
Super-K, have studied the problem, but because they are not equipped to distinguish between the various solar-generated neutrinos, the results have been inconclusive.

The SNO, a joint project between Canada, the United States and Britain, has perhaps the greatest chance of resolving the issue. Located at the bottom of one of North America's deepest mines, 300 kilometres north of Toronto, the SNO's detector is a spherical vat 12 metres across, filled with 1,000 tonnes of heavy water and lined with 10,000 PMTs. Heavy water contains deuterium - an isotope of hydrogen that has a neutron as well as a proton in its nucleus.

Most Cerenkov radiation observed at other detectors comes from interactions between electron neutrinos and either neutrons or electrons. But the SNO's deuterium atoms allow it to detect another type of collision which is more sensitive to the other neutrino flavours. Incoming neutrinos - electron, muon or tau - can, very occasionally, split the proton and neutron in deuterium apart. Gamma rays are generated if the neutron released by this fission is subsequently captured by another deuterium nucleus, and the SNO's PMTs can detect this radiation.

This ability to catch all three types of neutrinos is the SNO's big advantage. By comparing the numbers of each type of solar neutrino arriving at Earth with the number expected from theories of solar physics, the SNO should be able to settle the neutrino flavourswitching issue. The SNO team should have a major announcement within months, says Art McDonald, the observatory's director.

Other physicists are studying neutrinos produced by artificial sources such as nuclear reactors and particle accelerators. Researchers using these sources have more

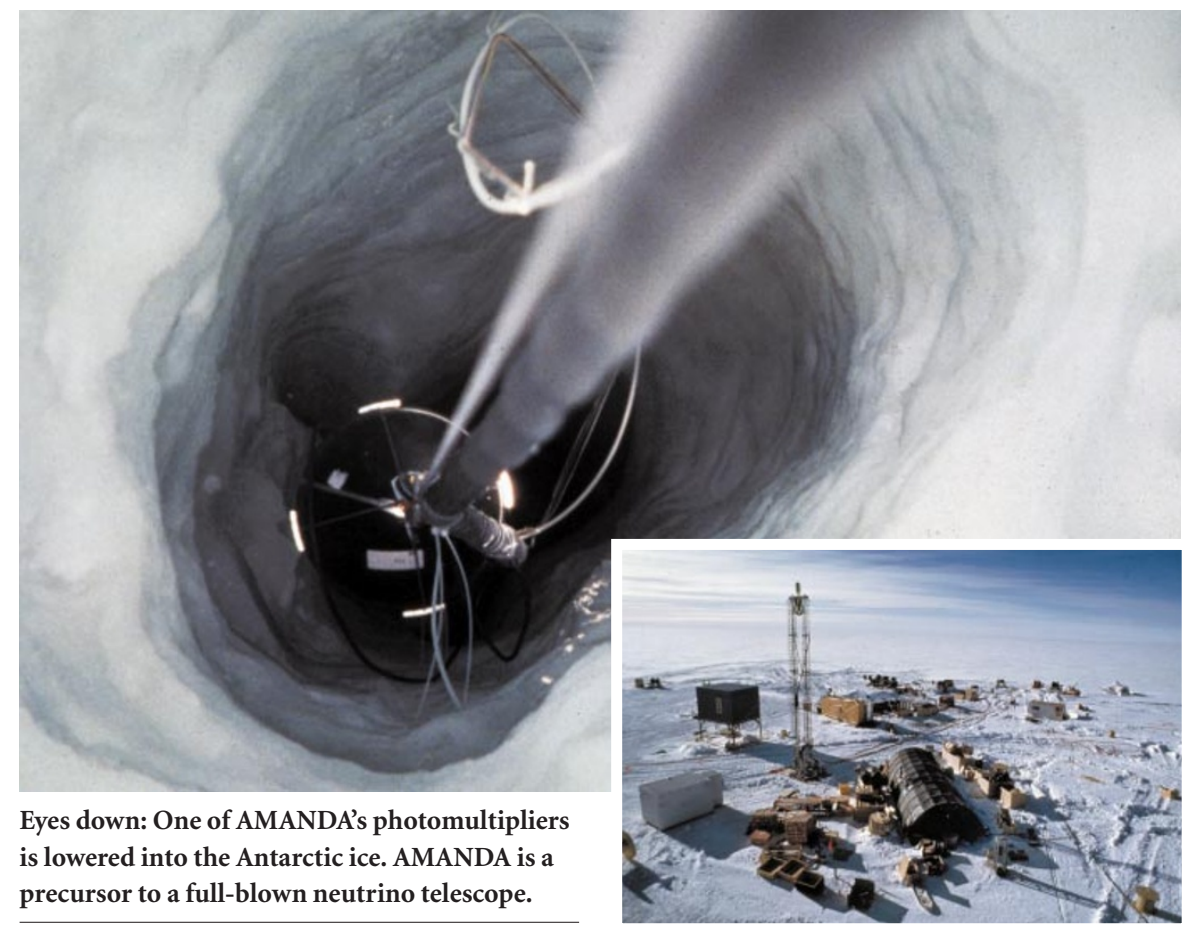




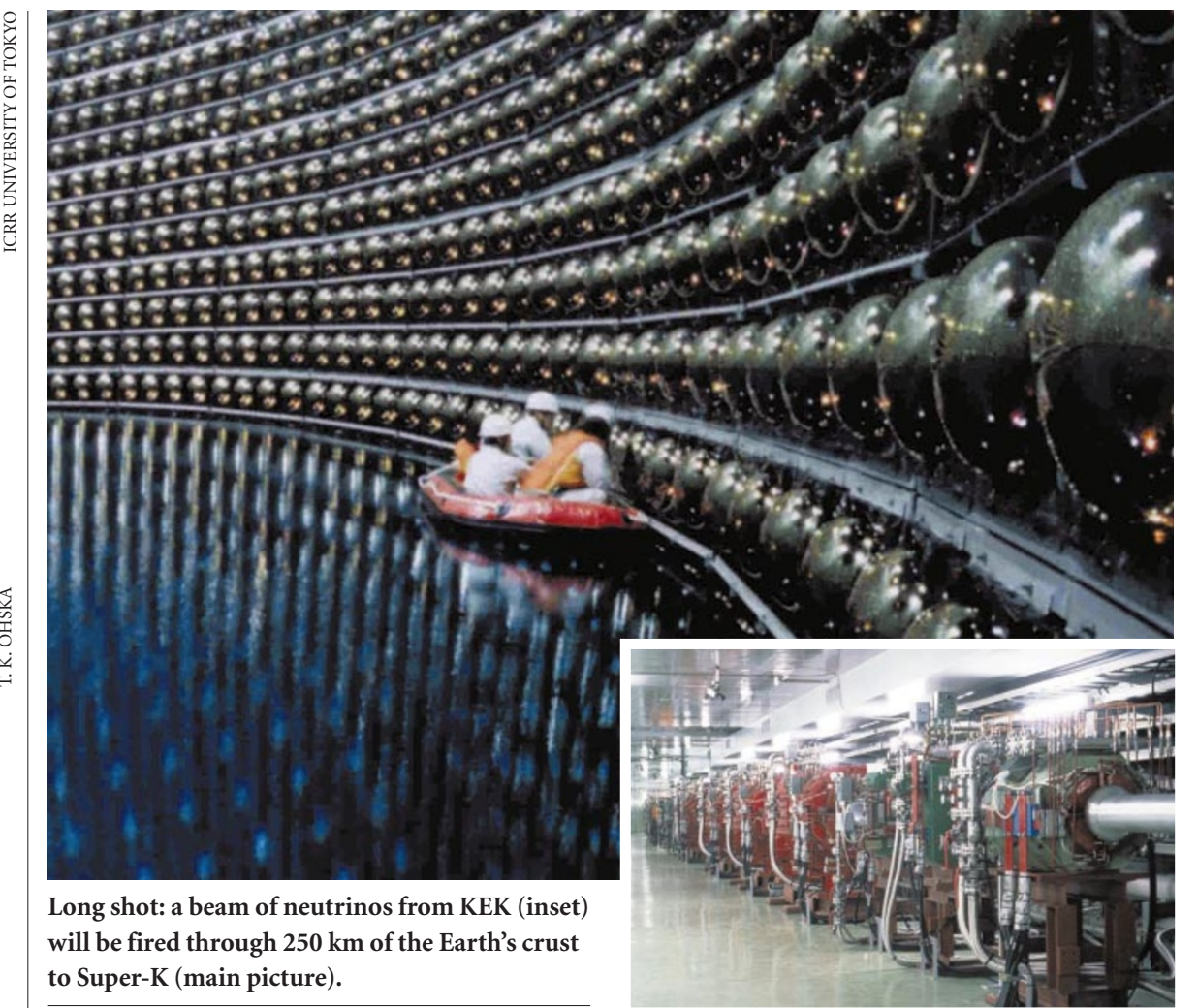

- information about how the neutrinos started out - where they came from, their energy and their total numbers. This allows them to look for oscillations under more controlled conditions.

In Japan, physicists at the KEK facility the High Energy Accelerator Research Organization - near Tokyo are using a particle accelerator to produce a beam of muon neutrinos. This is aimed through the Earth's crust towards Super-K, $250 \mathrm{~km}$ to the west. Physicists can estimate how many muon neutrinos Super-K should detect over long periods of time. After two years, says Totsuka, it has seen 28 neutrinos ${ }^{5}$. The theory says the detector should have seen 38 , suggesting that some of the muon neutrinos are turning into undetectable tau neutrinos on the way. The SuperK team says that the rate of switching inferred from the beam experiments is consistent with atmospheric neutrino studies. By July, says Totsuka, the team should have doubled the amount of data collected, and will publish more definitive results within a few years.

Two other similar experiments are in the planning stage. In Europe, physicists are going to fire a beam of neutrinos from CERN, the European Laboratory for Particle Physics near Geneva, through the Alps to Italy's Gran Sasso National Laboratory near Rome, $730 \mathrm{~km}$ away. The project should start collecting data in 2005. Rival physicists in the United States hope that the MINOS (Main Injector Neutrino Oscillation Search) project - a 725-km neutrino beam from Fermilab to the Soudan mine in Minnesota - will start producing results a year earlier.

Another 'controlled conditions' ap- proach will take advantage of the neutrinos produced in nuclear power plants. Later this year, a project called KamLAND (Kamioka Liquid Scintillator Antineutrino Detector) will start trying to detect antineutrinos, the antimatter equivalent to neutrinos, emitted by Japan's 51 nuclear power reactors, as well as solar neutrinos. This US-Japanese collaboration is housed in the same mine as Super$\mathrm{K}$, but uses a detector based on a liquid scintillator, a chemical that luminesces when low-energy neutrinos and antineutrinos pass through it.

\section{The hole picture}

Astronomers are also eager to get in on the neutrino detection act. They want to exploit neutrinos as a tool for studying some of the most energetic phenomena in the cosmos. As they travel through space, light and radio waves can be scattered or absorbed by gas and dust, but neutrinos carry information from their source to the Earth almost undisturbed, so astronomers can use them to study the farthest reaches of the Universe.

The dream of an operational neutrino telescope came a step closer in March, when the Antarctic Muon and Neutrino Detector Array (AMANDA) in Antarctica reported spottiing its first atmospheric neutrinos ${ }^{6}$. Buried in the ice some two kilometres below the South Pole, AMANDA consists of nearly 700 PMTs arranged in a huge cylinder, half a kilometre high and 200 metres in diameter. AMANDA is actually 'looking' downwards, searching for neutrinos that have travelled through the Earth's core. Like the layers of rock above the subterranean detectors, this screens out other subatomic particles. AMANDA detects fast-moving muons produced by collisions between highly energetic neutrinos and protons or neutrons. Other detectors miss the Cerenkov radiation produced by these muons because they move so quickly that they leave the detector before emitting useful amounts of light, but AMANDA's huge size allows it to spot them.

Innovative as AMANDA is, it is really only a stepping-stone towards a much larger cosmic neutrino detector, says Francis Halzen, part of the University of Wisconsin-Madison team that is leading the collaboration of European and US institutions behind the project. The partners are already drawing up plans for a much larger detector, dubbed IceCube. This should be able to detect neutrinos from cosmic sources such as black holes, quasars, supernovas and gamma-ray bursts. Bahcall likens AMANDA to a Model T Ford, and says: "IceCube will be the real Cadillac."

Physicists plan to use IceCube to study what happens to material approaching the event horizon of a black hole - the point of no return for the matter and light that the black hole is consuming. Clouds of hot swirling gas and dust around the black hole block the gaze of conventional telescopes, but neutrinos stream straight through, giving physicists an insight into this previously hidden region.

Addressing such mysteries should keep astrophysicists busy, but the most profound repercussions of the new era of neutrino studies could be for the standard model. If neutrinos do have mass, it will be the theory's first serious empirical challenge. "The finite neutrino mass is the first observational result beyond the standard model," says Totsuka.

If Super-K's initial results are backed up by the SNO and other detectors, the standard model will have to be corrected. And few physicists will be sorry. "The standard model is a paradox, because it's enormously successful in terms of describing a huge amount of data, but it has some features which are ugly - like having a whole bunch of arbitrary parameters," says Bahcall. Firm data on neutrino mass could help eliminate the ugliness and point the way to a more elegant theory. Dan Falk is a freelance writer based in Toronto.

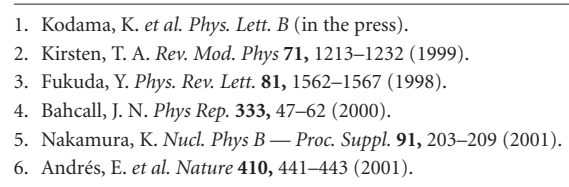

\section{Web links}

Sudbury Neutrino Observatory

http://www.sno.phy.queensu.ca

Super-Kamiokande

http://www-sk.icrr.u-tokyo.ac.jp/doc/sk

AMANDA

http://amanda.berkeley.edu/amanda/amanda.html IceCube

http://pheno.physics.wisc.edu/icecube 